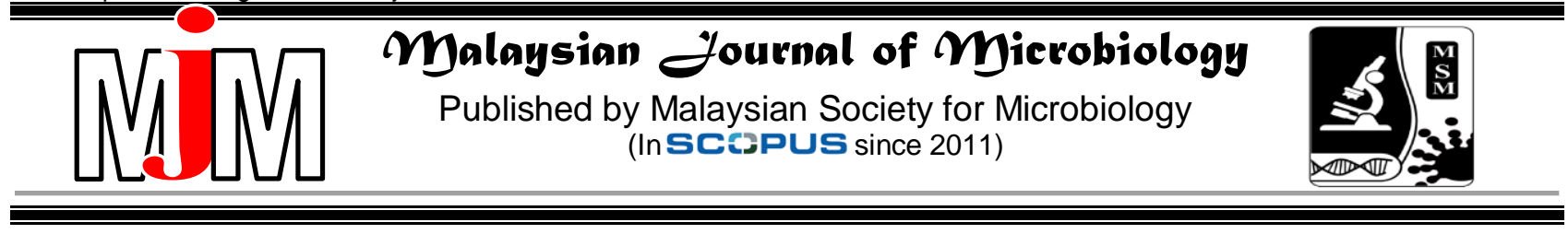

\title{
Distribution and prevalence of antibiotic resistant bacteria in fish farms in East Malaysia
}

\author{
Samuel Lihan ${ }^{1 *}$, Nurul Asyiqin Jamil², Mohd Azizul Hafiz Jamian ${ }^{3}$, Toh Seng Chiew², Olaide Olawunmi Ajibola², \\ Sabella Justin ${ }^{2}$, Flonia Benet ${ }^{2}$ and Lee Nung Kion ${ }^{4}$ \\ ${ }^{1}$ Institute of Biodiversity and Environmental Conservation, Universiti Malaysia Sarawak, 94300 Kota Samarahan, \\ Sarawak, Malaysia. \\ ${ }^{2}$ Faculty of Resource Science and Technology, Universiti Malaysia Sarawak, 94300 Kota Samarahan, Sarawak, \\ Malaysia. \\ ${ }^{3}$ Faculty of Social Science and Humanities, Universiti Malaysia Sarawak, 94300 Kota Samarahan, Sarawak, Malaysia. \\ ${ }^{4}$ Faculty of Cognitive Sciences and Human Development, Universiti Malaysia Sarawak, 94300 Kota Samarahan, \\ Sarawak, Malaysia. \\ Email: Isamuel@unimas.my
}

Received 13 August 2019; Received in revised form 2 March 2020; Accepted 12 March 2020

\begin{abstract}
Aims: Aquaculture has grown tremendously in Malaysia over the past decades. However, guaranteeing aquaculture sustainability is a big challenge in terms of maintaining continuous output with a safe environment. Furthermore, the cultured species should be free from antibiotic resistance bacterial and antibiotic residue. This study aimed to monitor the existence and prevalence of antibiotic resistant bacteria associated with aquaculture farms in Sarawak.

Methodology and results: Samples of water, sediment and fish were collected from five aquaculture farms within Sarawak. The samples were plated on trypticase soy agar and incubated at $28{ }^{\circ} \mathrm{C}$ for $24 \mathrm{~h}$. A total of 204 bacterial isolates were isolated and analysed by $(\mathrm{GTG})_{5}$-fingerprinting to determine genetic similarity among the bacterial isolates, so that representatives could be selected from similar clonal isolates. Based on the (GTG) $)_{5}$ profiles, 50 representative isolates were chosen for species identification using 16S rRNA sequencing. The identified bacteria were tested against 25 antibiotics using standard disk diffusion method. The 16S rRNA analysis revealed that the isolates constitute of 14 genera of bacteria including Bacillus (38\%), Exiguobacterium (16\%), Enterobacter (14\%), Aeromonas (6\%), Acinetobacter (4\%), Citrobacter (4\%), Staphylococcus (4\%), Achromobacter (2\%), Chitinophaga (2\%), Fictibacillus (2\%), Plesiomonas (2\%), Pseudomonas (2\%), Pseudoxanthomonas (2\%) and Stenotrophomonas (2\%). The antibiotic resistance analysis revealed that the highest percentage of resistance was recorded against streptomycin (75.0\%), followed by ampicillin (66.0\%), ceftriaxone (50.0\%), rifampin (43.3\%), aztreonam (36.8\%) and ceftazidime (31.6\%). Resistance to more than two antibiotics was observed in $40.0 \%$ of isolates with an overall multiple antibiotic resistant (MAR) index ranging from 0 to 0.79 .

Conclusion, significant and impact of study: The variability of antibiotic resistance patterns exhibited by different bacterial species suggests a dependence on selective pressures exhibited in different geographical locations. Our results show that the occurrence of MAR bacteria in an aquaculture environment with unknown history of antibiotics usage in the aquaculture system is possible, indicating a need to continuously monitor the presence of antibiotic resistant bacteria in the aquaculture system.
\end{abstract}

Keywords: Aquaculture, bacteria, 16S rRNA, antibiotic resistant

\section{INTRODUCTION}

Since the first antibiotic discovery by Alexander Fleming in 1928, many different classes of antibiotics have been developed and concurrently bacteria have slowly developed resistance towards those antimicrobial agents (Paulson et al., 2016). Now, the phenomenon of antibiotic resistance is a global problem in every sector including aquaculture and agriculture (Radhouani et al., 2014; Done et al., 2015). In Malaysia, multiple resistance bacteria and resistance genes have been reported to be present in the aquaculture and its surrounding environments (Samuel et al., 2011; Abdullahi et al., 2013; Kui Soon et al., 2014; Ng et al., 2014; Seng Chiew et al., 2018).

There have been continuous efforts in searching for 
potential sources of new antimicrobial agents by testing compounds from local natural resources (Samuel et al., 2014a; Samuel et al., 2014b; Farith et al., 2015). However, very few of the antimicrobial agents have been successfully developed because of difficulties in identifying and isolating the exact compounds with effective activity against bacteria (Singh, 2014; Swamy and Rudramurthy, 2016).

The reliance on antibiotics to combat bacterial diseases in the rapidly growing aquaculture industry is unavoidable (Bostock et al., 2010; Cabello et al., 2013). The use of antibiotics in the aquaculture industry is mainly for the purpose of treatment, control and prevention of diseases as well as for promoting the growth of cultured species (Phillips et al., 2004; Bush et al., 2011; Romero et al., 2012).

However, it is highly controversial that either the emergence of resistance bacteria is attributed to anthropogenic activities or as a result of intrinsic resistance and gene transfer among environmental bacteria (Bhullar et al., 2012). There has been growing evidence that antibiotic resistant bacteria (ARB) carries antibiotic resistance genes (ARGs) by mobile genetic elements such as, integrons and plasmids that have shown to be shared between aquatic bacteria and terrestrial animals and human pathogen (Cabello et al., 2013; Cantas et al., 2013). These genes movement allows a bacterium to build on existing adaptations to better adapt to the changing environment (Perry and Wright, 2013).

Malaysian brackish water aquaculture displayed a considerable growth in production by $6.7 \%$ (324.3 thousand tonnes) in 2017 and a slight decline in freshwater aquaculture by $0.8 \%$ (102.5 thousand tonnes) against the preceding year (Department of Statistic Malaysia, 2018). The existing aquaculture industry is, however, associated with an increasing number of large farms, high density of fish and poor sanitary conditions which could only lead to greater levels of resistance in the human commensal microbiota (Schmidt et al., 2000; Barton and Fløysand, 2010; Deng et al., 2016). This has become a global problem because the misuse of antibiotics in aquaculture has been identified to drives the emergence of antibiotic resistant bacteria (ARB) and led to an unwelcoming implication to the public health (Schmidt et al., 2000; Heuer et al., 2009; Deng et al., 2016; Patil et al., 2016; Paulson et al., 2016).

Thus, there is a need for surveillance on the use of antibiotics in aquaculture with a comprehensive regulatory framework for the registration of antibiotics drug, as current standards vary widely from one country to another (Cabello et al., 2013; Watts et al., 2017). An active enforcement by the health sector is of vast importance to ensure its safety and effectiveness. It is important that people working in the aquaculture industry learn how to use antibiotics in such a way that maximize their efficacy while minimizing the increased frequencies of resistant variants as the consequence of the antibiotics usage (Smith, 2008).

A clear view on the development and spread of resistance in aquaculture to protect the humans, animals, and ecosystem can be achieved by a better understanding of antibiotic resistance (AR) ecology through characterisation of antibiotic resistant bacteria and the ecology of antibiotic resistance prevalence in an aquaculture environment. Therefore, the objectives of this study are to isolate bacteria from the aquaculture and its environment and to determine the extent to which they are resistant to the commonly used antibiotics.

\section{MATERIALS AND METHODS}

\section{Sampling sites}

Five aquaculture farms located within the southern part of Sarawak, East Malaysia, were selected for the sampling in this study. The location of the farms on the map is shown in Figure 1. The global positioning system (GPS) coordinates of the farms were; Farm 1 (1 ${ }^{\circ} 26^{\prime} 57.80^{\prime \prime} \mathrm{N}$ $110^{\circ} 24^{\prime} 21.30^{\prime E}$ ), Farm 2 (132'53.43" N 110³2'53.99"E), Farm 3 (1'24'02.40" N 110'19'50.60"E), Farm 4 $\left(1^{\circ} 26^{\prime} 59.5^{\prime \prime} \mathrm{N} 110^{\circ} 10^{\prime} 12.0^{\prime \prime} \mathrm{E}\right)$, and Farm $5\left(1^{\circ} 26^{\prime} 09.6^{\prime \prime} \mathrm{N}\right.$ $\left.110^{\circ} 10^{\prime} 10.0 " \mathrm{E}\right)$.

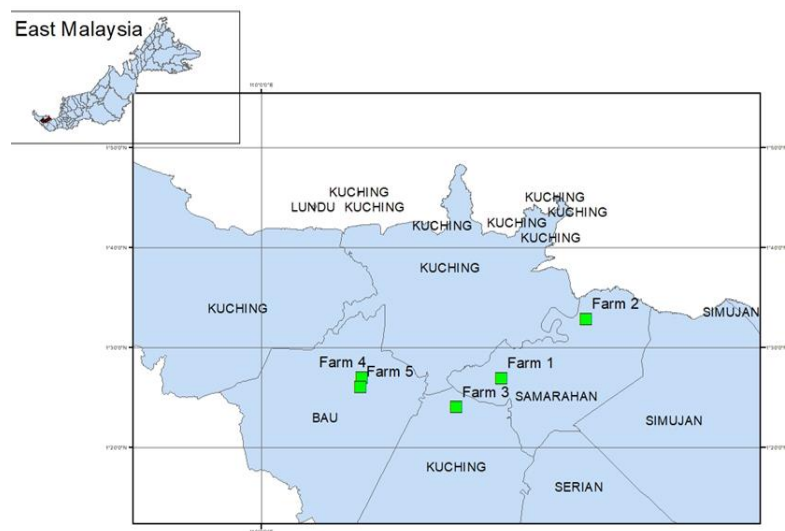

Figure 1: Sampling locations of five different farms in northern part of East Malaysia.

\section{Sample processing and bacterial isolation}

Samples of sediment, water and fish collected from the farms were processed according to the method described by Huys (2003). After the sample processing, the samples were plated on duplicate tryptone soy agar (Oxoid, USA) and incubated at $28^{\circ} \mathrm{C}$ for 18 to $24 \mathrm{~h}$. Bacterial colonies grown on the agar were randomly picked, purified and stocked in glycerol. Two hundred and four bacteria were isolated and kept in glycerol stock in $-20^{\circ} \mathrm{C}$ freezer.

\section{Bacterial characterization and identification}

Pure bacterial colonies were analysed for their genetic differences using (GTG) $)_{5}-\mathrm{PCR}$. Based on the (GTG) $)_{5}$ $\mathrm{PCR}$ profile as shown in Figure 2, a representative of fifty isolates was selected and identified using 16S rRNA gene 


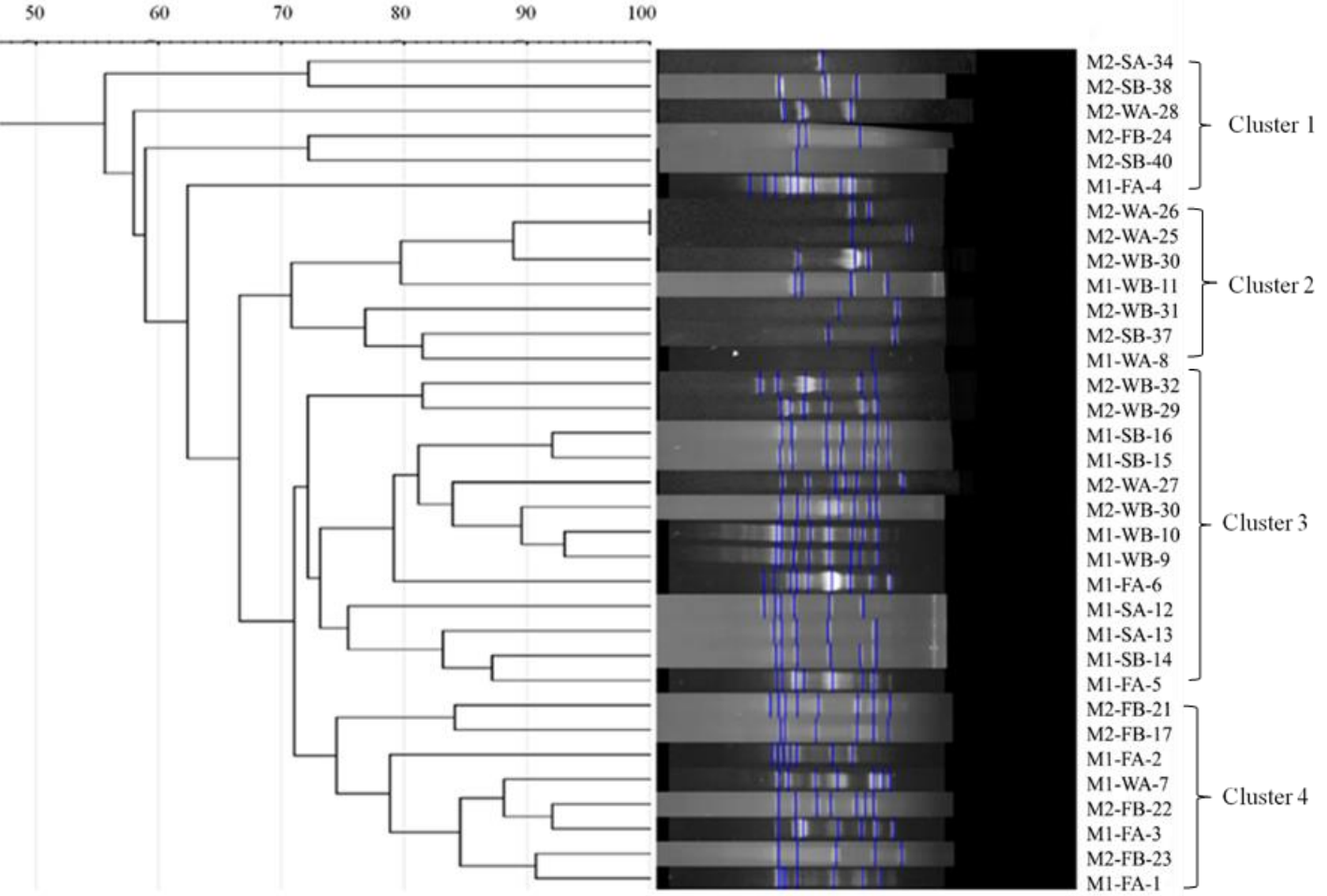

Figure 2: Representative profile and dendrogram of (GTG) $)_{5}-\mathrm{PCR}$ of the bacterial isolated from Farm 1. Dendrogram based on Dice similarity method and UPGMA linkage of (GTG) $)_{5}$ PCR fingerprints obtained from bacterial isolates. Cluster 1: M2-SA-34, M2-SB-38, M2-WA-28, M2-FB-24, M2-SB-40, M1-FA-4; Cluster 2: M2-WA-26, M2-WA25, M2-WB-30, M1-WB-11, M2-WB-31, M2-SB-37, M1-WA-8; Cluster 3: M2-WB-32, M2-WB-29, M1-SB-16, M1-SB-15, M2-WA-27, M2-WB-30, M1-WB-10, M1-WB-9, M1-FA-6, M1-SA-12, M1-SA-13, M1-SB-14, M1-FA-5; Cluster 4: M2-FB21, M2-FB-17, M1-FA-2, M1-WA-7, M2-FB-22, M1-FA-3, M2-FB-23, M1-FA-1.

sequencing with $27 \mathrm{~F} \quad$ (5'CAGGCCTAACACATGCAAGTC- 3') and 519R (5'GWATTACCGCGGCKGCTG - 3') primers in ancinguez-Palomares et al. (2007). Sequencing of the PCR product was carried out by First Base Laboratories (Selangor, Malaysia). The 16S rRNA sequence data were compared with available sequence data in the GenBank using BLAST.

\section{Antibiotic susceptibility test}

Fifty environmental isolates from 14 different genera were tested for antibiotic susceptibility using the disk diffusion method according to the recommendations of the CLSI (2017). All isolates were grown in Mueller-Hinton Broth (HiMedia, India) and then swabbed evenly onto the surface of Mueller-Hinton agar (HiMedia, India) plates. The plates were dried for 2-5 min before an antibiotic disk was placed on the agar surface using sterile forceps. The plates were then incubated at $28{ }^{\circ} \mathrm{C}$ for $18-20 \mathrm{~h}$. Twentyfive antibiotics selected for the test were amikacin (AK, 30 $\mu \mathrm{g})$, gentamicin $(\mathrm{CN}, 10 \mu \mathrm{g})$, kanamycin $(\mathrm{KA}, 30 \mu \mathrm{g})$, streptomycin (S, $10 \mu \mathrm{g})$, doxycycline (DO, $30 \mu \mathrm{g}$ ), tetracycline (TE, $30 \mu \mathrm{g})$, penicillin $(\mathrm{P}, 10 \mu \mathrm{g})$, piperacillin (PRL, $100 \mu \mathrm{g}$ ), ampicillin (AMP, $10 \mu \mathrm{g}$ ), chloramphenicol $(\mathrm{C}, 30 \mu \mathrm{g})$, levofloxacin (LEV, $5 \mu \mathrm{g})$, norfloxacin (NOR, 10 $\mu \mathrm{g}$ ), ciprofloxacin (CIP, $5 \mu \mathrm{g}$ ), nalidixic acid (NA, $30 \mu \mathrm{g})$, rifampin (RD, $5 \mu \mathrm{g})$, erythromycin $(\mathrm{E}, 15 \mu \mathrm{g})$, trimethoprim-sulfamethoxazole (SXT, 1.25/23.75 $\mu \mathrm{g}$ ), cefepime (FEP, $30 \mu \mathrm{g}$ ), ceftazidime (CAZ, $30 \mu \mathrm{g})$, meropenem (MEM, $10 \mu \mathrm{g})$, ertapenem (ETP, $10 \mu \mathrm{g}$ ), imipenem (IMP, $10 \mu \mathrm{g}$ ), aztreonam (ATM, $30 \mu \mathrm{g}$ ), ceftriaxone (CRO, $30 \mu \mathrm{g})$ and cephalothin (KF, $30 \mu \mathrm{g})$. Cultures of E. coli ATCC 25922 and Staphylococcus aureus ATCC 25923 were included as controls in the susceptibility testing. The assessment of the bacterial susceptibility to the twenty-five different antibiotics is shown in Table 1.

\section{MAR index assessment}

The multiple antibiotic resistance (MAR) index analysis was employed in accordance to Krumperman (1983) formula, where the number of antibiotics to which the bacterium was resistant to was divided by the number of antibiotics to which the isolates were tested upon. 
Malays. J. Microbiol. Vol 16(4) 2020, pp. 263-274

DOI: http://dx.doi.org/10.21161/mjm. 190522

Table 1: Antibiogram of aquatic bacteria from the fish farms and their MAR index.

\begin{tabular}{|c|c|c|c|c|c|c|c|c|c|c|c|c|c|c|c|c|c|c|c|c|c|c|c|c|c|c|c|}
\hline \multirow{2}{*}{ 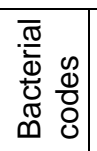 } & \multirow{2}{*}{ 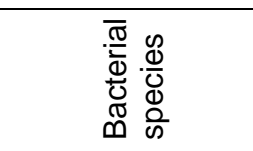 } & \multicolumn{25}{|c|}{ Antibiotics } & \multirow[b]{2}{*}{ 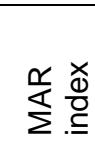 } \\
\hline & & $\stackrel{y}{<}$ & $\stackrel{\varpi}{\models}$ & 음 & Z & $a$ & 0 & 岃 & $\stackrel{p}{\simeq}$ & 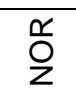 & ш & $\frac{n}{U}$ & $\frac{\vec{\alpha}}{\alpha}$ & 文 & 邑 & $\sum_{\Sigma}$ & $\frac{N}{\delta}$ & $\S$ & $\S$ & $\omega$ & $\sum_{<}^{0}$ & $\underset{⿱ 亠 乂}{\sum}$ & $\frac{n}{w}$ & $\stackrel{\varrho}{\Sigma}$ & 㟔 & $\begin{array}{l}\stackrel{0}{1} \\
\text { O }\end{array}$ & \\
\hline $\begin{array}{l}\text { PM } \\
249\end{array}$ & Bacillus sp. & $S$ & S & $\mathrm{S}$ & $\mathrm{S}$ & $\mathrm{R}$ & $\mathrm{S}$ & $\mathrm{S}$ & $\mathrm{R}$ & $\mathrm{S}$ & $\mathrm{R}$ & $\mathrm{S}$ & NT & NT & NT & NT & NT & NT & NT & NT & NT & NT & NT & NT & NT & NT & 0.27 \\
\hline $\begin{array}{l}\text { LT } \\
26\end{array}$ & Bacillus cereus & $S$ & $S$ & $S$ & $S$ & $S$ & $S$ & $S$ & I & $S$ & $S$ & $S$ & NT & NT & NT & NT & NT & NT & NT & NT & NT & NT & NT & NT & NT & NT & 0.09 \\
\hline $\begin{array}{l}\mathrm{LT} \\
17\end{array}$ & Bacillus pumilus & $\mathrm{S}$ & S & $S$ & $S$ & $S$ & $S$ & $S$ & I & $S$ & $\mathrm{~S}$ & $\mathrm{~S}$ & NT & NT & NT & NT & NT & NT & NT & NT & NT & NT & NT & NT & NT & NT & 0.09 \\
\hline $\begin{array}{l}J \\
200\end{array}$ & Bacillus cereus & $\mathrm{S}$ & $\mathrm{R}$ & $\mathrm{S}$ & I & $\mathrm{R}$ & S & S & $\mathrm{R}$ & $\mathrm{S}$ & $\mathrm{S}$ & $\mathrm{S}$ & NT & NT & NT & NT & NT & NT & NT & NT & NT & NT & NT & NT & NT & NT & 0.36 \\
\hline $\begin{array}{l}J \\
187\end{array}$ & Bacillus sp. & $S$ & $S$ & $S$ & $S$ & $S$ & $S$ & $S$ & I & $S$ & I & $S$ & NT & NT & NT & NT & NT & NT & NT & NT & NT & NT & NT & NT & NT & NT & 0.18 \\
\hline $\begin{array}{l}\mathrm{LT} \\
18\end{array}$ & Bacillus pumilus & $S$ & $S$ & $S$ & $S$ & $S$ & $S$ & $S$ & $S$ & $S$ & $S$ & $S$ & NT & NT & NT & NT & NT & NT & NT & NT & NT & NT & NT & NT & NT & NT & 0 \\
\hline $\begin{array}{l}\text { PM } \\
207\end{array}$ & Bacillus sp. & $\mathrm{S}$ & $S$ & $S$ & $S$ & $\mathrm{R}$ & $S$ & $S$ & I & $S$ & I & $S$ & NT & NT & NT & NT & NT & NT & NT & NT & NT & NT & NT & NT & NT & NT & 0.27 \\
\hline $\begin{array}{l}\mathrm{LT} \\
21\end{array}$ & $\begin{array}{l}\text { Bacillus } \\
\text { aquimaris }\end{array}$ & $S$ & $\mathrm{~S}$ & $S$ & $S$ & $S$ & $S$ & $S$ & $S$ & $S$ & $S$ & $S$ & NT & NT & NT & NT & NT & NT & NT & NT & NT & NT & NT & NT & NT & NT & 0 \\
\hline $\begin{array}{l}\text { LT } \\
40\end{array}$ & $\begin{array}{l}\text { Exiguobacterum } \\
\text { profundum }\end{array}$ & $S$ & $S$ & $S$ & $S$ & $S$ & $S$ & $S$ & $S$ & $S$ & $S$ & $S$ & NT & NT & NT & NT & NT & NT & NT & NT & NT & NT & NT & NT & NT & NT & 0 \\
\hline $\begin{array}{l}\text { PM } \\
185\end{array}$ & Bacillus indicus & $S$ & $S$ & $S$ & $S$ & $S$ & $S$ & $S$ & I & $S$ & $S$ & $S$ & NT & NT & NT & NT & NT & NT & NT & NT & NT & NT & NT & NT & NT & NT & 0.09 \\
\hline $\begin{array}{l}\text { SM } \\
103\end{array}$ & $\begin{array}{l}\text { Staphylococcus } \\
\text { sp. }\end{array}$ & $S$ & 1 & $S$ & $S$ & $S$ & $S$ & $S$ & $S$ & $S$ & $S$ & $S$ & NT & NT & NT & NT & NT & NT & NT & NT & NT & NT & NT & $\mathrm{NT}$ & NT & NT & 0.09 \\
\hline $\begin{array}{l}\text { SM } \\
94\end{array}$ & $\begin{array}{l}\text { Bacillus } \\
\text { zhangzhouensis }\end{array}$ & $S$ & $S$ & $S$ & $S$ & $S$ & $S$ & $S$ & $S$ & $S$ & $S$ & $S$ & NT & NT & NT & NT & NT & NT & NT & NT & NT & NT & NT & NT & NT & NT & 0 \\
\hline $\begin{array}{l}\mathrm{LT} \\
63\end{array}$ & $\begin{array}{l}\text { Exiguobacterium } \\
\text { profundum }\end{array}$ & $S$ & S & $S$ & $S$ & $S$ & $S$ & $S$ & $\mathrm{~S}$ & S & $\mathrm{R}$ & $\mathrm{S}$ & NT & NT & NT & NT & NT & NT & NT & NT & NT & NT & NT & NT & NT & NT & 0.09 \\
\hline $\begin{array}{l}\text { LT } \\
64\end{array}$ & $\begin{array}{l}\text { Exiguobacterium } \\
\text { aurantiacum }\end{array}$ & $S$ & $S$ & $S$ & $S$ & $S$ & $S$ & $S$ & $S$ & $\mathrm{R}$ & $\mathrm{R}$ & $S$ & NT & NT & NT & NT & NT & NT & NT & NT & NT & NT & NT & NT & NT & NT & 0.18 \\
\hline $\begin{array}{l}\text { SM } \\
91\end{array}$ & Bacillus cereus & $S$ & $S$ & $S$ & $S$ & $\mathrm{R}$ & $S$ & $S$ & $S$ & $S$ & $S$ & $S$ & NT & NT & NT & NT & NT & NT & NT & NT & NT & NT & NT & $\mathrm{NT}$ & NT & NT & 0.09 \\
\hline
\end{tabular}


Malays. J. Microbiol. Vol 16(4) 2020, pp. 263-274

DOI: http://dx.doi.org/10.21161/mjm. 190522

(Continued)

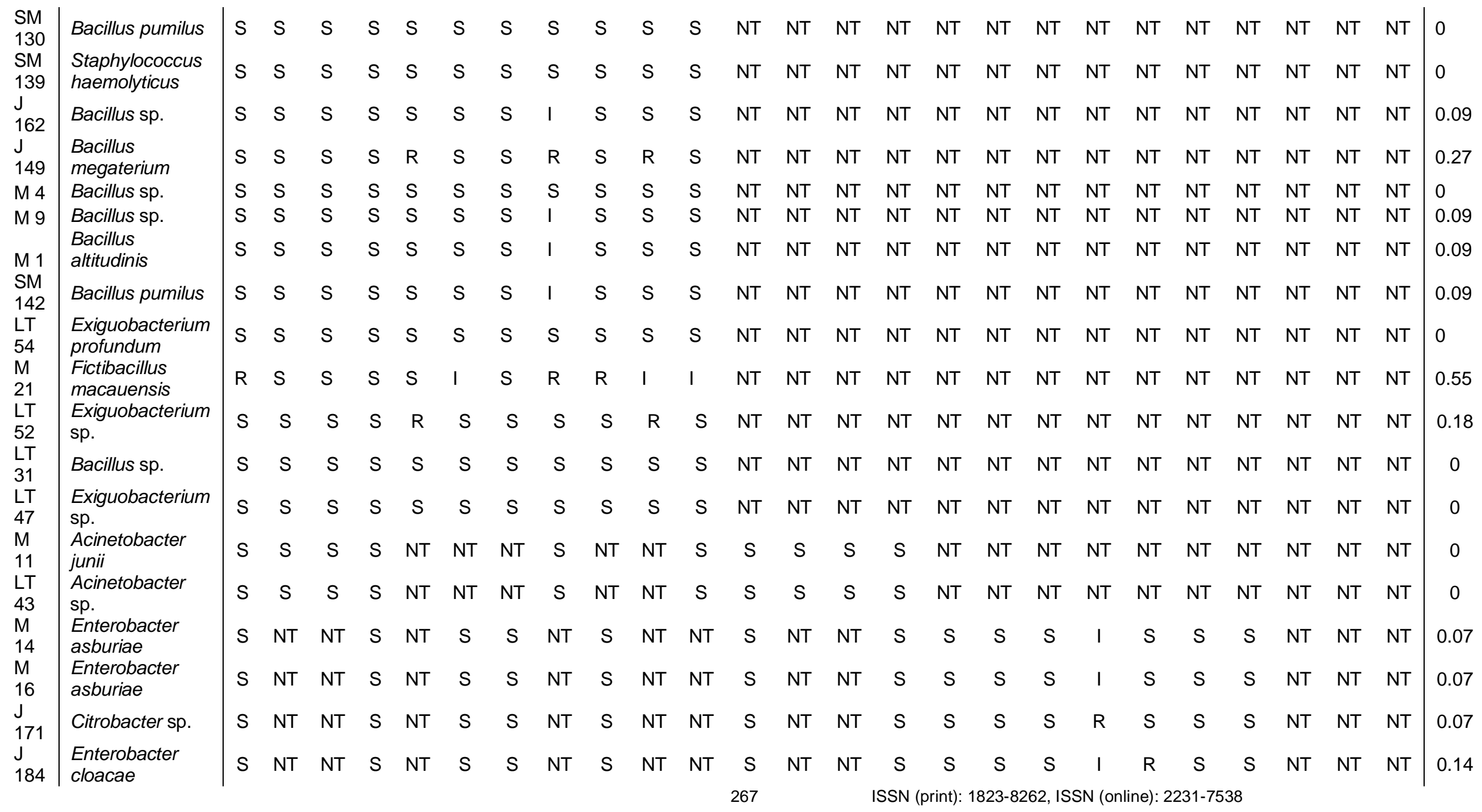


Malays. J. Microbiol. Vol 16(4) 2020, pp. 263-274

DOI: http://dx.doi.org/10.21161/mjm. 190522

(Continued)

\begin{tabular}{|c|c|c|c|c|c|c|c|c|c|c|c|c|c|c|c|c|c|c|c|c|c|c|c|c|c|c|c|}
\hline $\begin{array}{l}\mathrm{PM} \\
205\end{array}$ & Enterobacter sp. & $\mathrm{S}$ & NT & NT & $\mathrm{S}$ & NT & $\mathrm{S}$ & $\mathrm{S}$ & NT & $\mathrm{S}$ & NT & NT & $\mathrm{S}$ & NT & NT & 1 & $\mathrm{~S}$ & $\mathrm{~S}$ & $\mathrm{~S}$ & I & $\mathrm{R}$ & $\mathrm{S}$ & I & NT & NT & NT & 0.29 \\
\hline $\begin{array}{l}\mathrm{LT} \\
23\end{array}$ & Enterobacter sp. & $S$ & NT & NT & $\mathrm{S}$ & NT & $S$ & $\mathrm{~S}$ & NT & $\mathrm{S}$ & NT & NT & $\mathrm{S}$ & NT & NT & $\mathrm{S}$ & I & $\mathrm{S}$ & $S$ & $\mathrm{~S}$ & $\mathrm{R}$ & $S$ & $S$ & NT & NT & NT & 0.14 \\
\hline $\begin{array}{l}\mathrm{J} 2 \\
176\end{array}$ & Citrobacter sp. & $S$ & NT & NT & $\mathrm{S}$ & NT & $S$ & $S$ & NT & $S$ & NT & NT & $\mathrm{S}$ & NT & NT & $\mathrm{S}$ & $S$ & $S$ & $S$ & $\mathrm{~S}$ & $\mathrm{R}$ & $\mathrm{S}$ & $S$ & NT & NT & NT & 0.07 \\
\hline $\begin{array}{l}M \\
01\end{array}$ & Chitinophaga sp. & $\mathrm{R}$ & NT & NT & $\mathrm{R}$ & NT & $\mathrm{R}$ & $S$ & NT & I & NT & NT & $\mathrm{R}$ & NT & NT & $S$ & $\mathrm{R}$ & $\mathrm{R}$ & $\mathrm{S}$ & $\mathrm{R}$ & $\mathrm{R}$ & $\mathrm{R}$ & $\mathrm{R}$ & NT & NT & NT & 0.79 \\
\hline 151 & $\begin{array}{l}\text { Enterobacter } \\
\text { amnigenus }\end{array}$ & $S$ & NT & NT & $S$ & NT & $S$ & $S$ & NT & $S$ & $\mathrm{NT}$ & NT & $S$ & NT & NT & $S$ & $S$ & $S$ & $S$ & & $\mathrm{R}$ & $S$ & $S$ & NT & NT & NT & 0.14 \\
\hline 246 & Enterobacter sp. & $S$ & NT & NT & $S$ & NT & $S$ & $S$ & NT & $S$ & NT & NT & I & NT & NT & $S$ & $\mathrm{R}$ & $S$ & $S$ & $S$ & $S$ & $\mathrm{R}$ & $S$ & NT & NT & NT & 0.21 \\
\hline $\begin{array}{l}2 \mathrm{M} \\
216\end{array}$ & Enterobacter sp. & S & NT & NT & $S$ & NT & $S$ & $S$ & NT & $S$ & $\mathrm{NT}$ & NT & $S$ & NT & NT & S & $S$ & 1 & $S$ & I & $\mathrm{R}$ & $S$ & I & NT & NT & NT & 0.29 \\
\hline 8 & Enterobacter sp. & $S$ & NT & NT & $S$ & NT & $\mathrm{R}$ & $S$ & NT & $S$ & NT & NT & $S$ & NT & NT & $S$ & $S$ & $S$ & $S$ & $\mathrm{R}$ & $\mathrm{R}$ & $S$ & $S$ & NT & NT & NT & 0.21 \\
\hline $\begin{array}{l}\text { SM } \\
79\end{array}$ & $\begin{array}{l}\text { Pseudoxanthomonas } \\
\text { mexicana }\end{array}$ & NT & NT & NT & $\mathrm{R}$ & NT & NT & $S$ & NT & $\mathrm{NT}$ & $\mathrm{NT}$ & $S$ & I & NT & $R$ & $\mathrm{R}$ & $\mathrm{R}$ & NT & NT & NT & $\mathrm{NT}$ & $\mathrm{R}$ & NT & $\mathrm{R}$ & NT & NT & 0.78 \\
\hline $\begin{array}{l}J \\
152\end{array}$ & Achromobacter sp. & NT & NT & NT & $S$ & NT & NT & $S$ & NT & $\mathrm{NT}$ & $\mathrm{NT}$ & $S$ & $S$ & NT & $S$ & $S$ & $S$ & NT & NT & NT & NT & $\mathrm{R}$ & NT & $S$ & NT & NT & 0.11 \\
\hline $\begin{array}{l}\text { PM } \\
199\end{array}$ & Pseudomonas sp. & NT & NT & NT & $S$ & NT & NT & $S$ & NT & NT & NT & $S$ & $S$ & NT & $S$ & $S$ & $S$ & NT & NT & NT & NT & $S$ & NT & $S$ & NT & NT & 0 \\
\hline $\begin{array}{l}\mathrm{LT} \\
16\end{array}$ & $\begin{array}{l}\text { Stenotrophomonas } \\
\text { sp. }\end{array}$ & NT & NT & NT & NT & NT & NT & $S$ & NT & NT & NT & NT & NT & $S$ & NT & NT & NT & NT & NT & NT & NT & NT & NT & NT & NT & NT & 0 \\
\hline $\begin{array}{l}M \\
40\end{array}$ & Aeromonas veronii & S & $S$ & NT & $S$ & NT & $S$ & NT & NT & NT & NT & $S$ & NT & NT & NT & $S$ & $\mathrm{R}$ & NT & NT & NT & NT & $\mathrm{R}$ & NT & $S$ & $S$ & I & 0.27 \\
\hline $\begin{array}{l}\mathrm{PM} \\
257\end{array}$ & $\begin{array}{l}\text { Plesiomonas } \\
\text { shigelloides }\end{array}$ & 1 & I & NT & I & NT & $S$ & NT & NT & NT & NT & $S$ & NT & NT & NT & $S$ & $S$ & NT & NT & NT & NT & $S$ & NT & $S$ & $\mathrm{R}$ & $S$ & 0.36 \\
\hline $\begin{array}{l}\text { PM } \\
183\end{array}$ & Aeromonas veronii & $S$ & $S$ & NT & $S$ & NT & $S$ & $\mathrm{NT}$ & NT & NT & $\mathrm{NT}$ & $S$ & NT & NT & NT & $S$ & $S$ & NT & NT & NT & $\mathrm{NT}$ & $\mathrm{R}$ & NT & $S$ & $S$ & $S$ & 0.09 \\
\hline $\begin{array}{l}J \\
181\end{array}$ & Aeromonas jandaei & $\mathrm{R}$ & $S$ & NT & $S$ & NT & $S$ & $\mathrm{NT}$ & NT & NT & NT & $S$ & NT & NT & NT & $S$ & $\mathrm{R}$ & NT & NT & NT & $\mathrm{NT}$ & $\mathrm{R}$ & NT & $S$ & $S$ & $\mathrm{R}$ & 0.36 \\
\hline
\end{tabular}

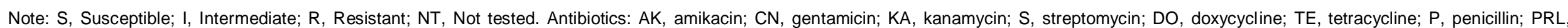

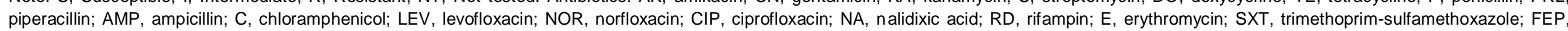
cefepime; CAZ, ceftazidime; MEM, meropenem; ETP, ertapenem; IMP, imipenem; ATM, aztreonam; CRO, ceftriaxone; KF, cephalothin. 


\section{RESULTS}

Antibiotics selection was dependent on the bacterial genera, as different bacterial groups may be intrinsically resistant to certain antibiotics which explained the unnecessary need to be tested against certain antibiotics classes. The list of bacterial isolates consisting 14 different genera of bacteria tested in this study is shown in Table 1.

The highest percentage of resistance recorded was against streptomycin (75.0\%), followed by ampicillin $(66.7 \%)$, ceftriaxone $(50.0 \%)$, rifampin (43.3\%), aztreonam (36.8\%) and ceftazidime (31.6\%) as shown in Table 2. In addition, the bacterial isolates showed susceptibility towards four antibiotics; doxycycline, levofloxacin, trimethoprim-sulfamethoxazole and nalidixic acid. A representative profile together with the dendrogram constructed out of $(\mathrm{GTG})_{5}-\mathrm{PCR}$ of the bacteria isolated from Farm 1 is shown in Figure 2. The dendrogram was constructed based on the Dice similarity method and UPGMA linkage of $(\mathrm{GTG})_{5}-\mathrm{PCR}$ fingerprints obtained from bacterial isolates.

Twenty-four different resistance patterns were found in this study as shown in Table 3 . Resistance to only one antibiotic was seen in $32.0 \%(16 / 50)$ of isolates. Out of all the isolates, $40.0 \%(20 / 50)$ were found to be multiple resistances. There were four resistance patterns shared by at least two or more bacteria. The variability of antibiotic resistance patterns exhibited by different bacterial species suggests selective pressures exhibit in the aqueous habitats which is in agreement with studies conducted by Lesley et al. (2011) and Kathleen et al. (2016).

Fisher's exact test was applied on Bacillus sp. which accounts for the majority of the isolates $(38 \%, n=19 / 50)$ to determine if there was any significant difference in the proportion of Bacillus between the sample sources (sediment, water, and fish) and the risk level from antibiotic contaminated areas (high $\geq 0.2$ or low $\leq 0.2$ ). Group sizes of Bacillus from the three-sample sources were unequal, where 10 Bacillus sp. were identified from the sediment, 5 isolates from water and 4 isolates from fish. Based on this study, there was no significant difference in proportions of Bacillus sp. from low or highrisk level from antibiotic contaminated areas in these three sources $(p=0.373)$. This finding is in agreement with Schmidt et al. (2000), where the resistance level between water and fish isolates showed no significant difference.

Table 2: Percentage of bacterial resistance to different antibiotics in the fish farms.

\begin{tabular}{lccc}
\hline Antibiotics & $\begin{array}{c}\text { Abbreviation } \\
\text { of antibiotics }\end{array}$ & Total of resistant isolates (total isolates tested) & $\begin{array}{c}\text { Percentage of } \\
\text { resistance } \\
(\%)\end{array}$ \\
\hline Streptomycin & S & $9(12)$ & 75.0 \\
Ampicillin & AMP & $8(12)$ & 66.7 \\
Ceftriaxone & CRO & $2(4)$ & 50.0 \\
Rifampin & RD & $13(30)$ & 43.3 \\
Aztreonam & ATM & $7(19)$ & 36.8 \\
Ceftazidime & CAZ & $6(19)$ & 31.6 \\
Erythromycin & E & $8(28)$ & 28.6 \\
Ertapenem & ETP & $3(12)$ & 25.0 \\
Cephalothin & KF & $1(4)$ & 25.0 \\
Penicillin & P & $6(28)$ & 21.4 \\
Cefepime & FEP & $1(5)$ & 20.0 \\
Piperacillin & PRL & $3(17)$ & 17.6 \\
Kanamycin & KA & $2(12)$ & 16.7 \\
Imipenem & IMP & $1(7)$ & 14.3 \\
Meropenem & MEM & $2(21)$ & 9.5 \\
Tetracycline & TE & $3(34)$ & 8.8 \\
Chloramphenicol & C & $3(38)$ & 8.8 \\
Amikacin & AK & $4(46)$ & 8.7 \\
Gentamycin & CN & $4(49)$ & 8.2 \\
Norfloxacin & NOR & $3(40)$ & 7.5 \\
Ciprofloxacin & CIP & $1(37)$ & 2.7 \\
Doxycycline & DO & $0(30)$ & 0.0 \\
Levofloxacin & LEV & $0(42)$ & 0.0 \\
Trimethoprim- & SXT & $0(3)$ & 0.0 \\
sulfamethoxazole & NA & $0(12)$ & 0.0 \\
Nalidixic Acid & & & \\
\hline & & &
\end{tabular}


Malays. J. Microbiol. Vol 16(4) 2020, pp. 263-274

DOI: http://dx.doi.org/10.21161/mjm.190522

Table 3: Resistant patterns and MAR index of aquaculture bacteria.

\begin{tabular}{|c|c|c|c|}
\hline MAR index & Resistant pattern & Isolates code & Percentage of isolate (\%) \\
\hline 0.79 & $\begin{array}{l}\text { NOR-AK-CN-C-PRL-CAZ-KA-S- } \\
\text { AMP-ATM-ETP }\end{array}$ & SM 101 & 2.0 \\
\hline 0.78 & PRL-CN-FEP-MEM-CAZ-ATM-IMP & SM 79 & 2.0 \\
\hline 0.55 & C-E-CIP-AK-RD-NOR & M 21 & 2.0 \\
\hline 0.36 & $\begin{array}{l}\text { CN-TE-P-RD } \\
\text { AK-TE-CN- KF } \\
\text { AK-CAZ-ATM-KF }\end{array}$ & $\begin{array}{l}\text { J } 200 \\
\text { PM } 257 \\
\text { J } 181\end{array}$ & 6.0 \\
\hline 0.29 & $\begin{array}{l}\text { MEM-S-ETP-AMP } \\
\text { KA-S-ETP-AMP }\end{array}$ & $\begin{array}{l}\text { PM } 205 \\
\text { PM } 216\end{array}$ & 4.0 \\
\hline 0.27 & $\begin{array}{l}\text { P-RD-E } \\
\text { CRO- CAZ-ATM }\end{array}$ & $\begin{array}{l}\text { PM 249, PM 207, J } 149 \\
\text { M40 }\end{array}$ & 8.0 \\
\hline 0.21 & $\begin{array}{l}\text { PRL-CAZ-ATM } \\
\text { C-PRL-AMP }\end{array}$ & $\begin{array}{l}\text { PM } 246 \\
\text { LT } 78\end{array}$ & 4.0 \\
\hline 0.18 & $\begin{array}{l}\text { RD-E } \\
\text { NOR-E } \\
\text { P-E }\end{array}$ & $\begin{array}{l}\text { J } 187 \\
\text { LT } 64 \\
\text { LT } 52\end{array}$ & 6.0 \\
\hline 0.14 & $\begin{array}{l}\text { S-AMP } \\
\text { CAZ-AMP }\end{array}$ & $\begin{array}{l}\mathrm{J} 184, \mathrm{~J} 151 \\
\text { LT } 23\end{array}$ & 6.0 \\
\hline 0.11 & ATM & $\mathrm{J} 152$ & 2.0 \\
\hline 0.09 & $\begin{array}{l}\text { RD } \\
\text { TE } \\
\text { E } \\
\text { P } \\
\text { ATM }\end{array}$ & $\begin{array}{l}\text { LT 26, LT17, PM 185, J } \\
162, \text { M 9, M 1, SM } 142 \\
\text { SM } 103 \\
\text { LT } 63 \\
\text { SM } 91 \\
\text { PM } 183\end{array}$ & 22.0 \\
\hline 0.07 & $\begin{array}{l}\mathrm{S} \\
\mathrm{AMP}\end{array}$ & $\begin{array}{l}\text { M 14, M 16, J } 171 \\
\text { J } 176\end{array}$ & 8.0 \\
\hline 0 & - & $\begin{array}{l}\text { LT 18, LT 21, LT 40, SM } \\
\text { 94, SM 130, SM 139, M } \\
\text { 4, LT 54, LT 31, LT 47, M } \\
\text { 11, LT 43, PM 199, LT } 16\end{array}$ & 28.0 \\
\hline
\end{tabular}

Note: AK, amikacin; CN, gentamicin; KA, kanamycin; S, streptomycin; DO, doxycycline; TE, tetracycline; P, penicillin; PRL, piperacillin; AMP, ampicillin; C, chloramphenicol; LEV, levofloxacin; NOR, norfloxacin; CIP, ciprofloxacin; NA, nalidixic acid; RD, rifampin; E, erythromycin; SXT, trimethoprim-sulfamethoxazole; FEP, cefepime; CAZ, ceftazidime; MEM, meropenem; ETP, ertapenem; IMP, imipenem; ATM, aztreonam; CRO, ceftriaxone; KF, cephalothin. 


\section{DISCUSSION}

The direct use of antibiotics in human health and the use of the antimicrobial agents in animal husbandry for growth promoters and disease treatment have been responsible for the significant rise in multiple antibiotic resistance with the potential for transfer of the organisms or of genetic material coding for resistance to humans. The antimicrobial resistance of bacteria in animals including fish reared in the aquaculture sector should be monitored continuously.

In this study, it was found that 16 out of 50 (32\%) representative environmental bacteria were resistant to only one of the antibiotics tested. Not surprisingly, antibiotic resistance in aquatic bacteria was found in the water sample as it is accepted as a mixing ground for gene exchange between environmental bacteria (Perry and Wright, 2013). It is, however, difficult to conclude that antibiotic resistance in the fish farms is attributed to anthropogenic activities only, as Chamosa et al. (2017) suggested that there could be a bidirectional flow of similar antimicrobial resistant bacteria and genes encountered in both the environment and human microbiota.

A high percentage of isolates $(74.0 \%, n=37 / 50)$ display MAR indices less than 0.2. According to Krumperman (1983), isolates originate from a lower antibiotic contaminated source showed MAR indices of 0.2 and below. This suggests that most isolates originate from a lower antibiotic contaminated source (Krumperman, 1983; Tanil et al., 2005), which in turn suggests a low or no history of usage of antibiotics in the aquaculture farms studied (Lesley et al., 2018). Similar results were obtained from a study conducted by Kathleen et al. (2016), whereby most isolates $(63.1 \%, n=$ 94) were found to be from lower antibiotic contaminated sources. The overall results indicated that MAR indices ranged from 0 to 0.79 , with the highest resistance seen in Chitinophaga sp. (Table 1). This bacterial species showed resistance to 11 out of the 14 antibiotics tested.

Table 2 shows the percentage of bacterial resistance to different antibiotics in the fish farms. A high percentage of resistance to streptomycin $(75 \% ; 9 / 12)$ and ampicillin $(66.7 \% ; 8 / 12)$ was observed among the aquacultural bacterial isolates. This observation is consistent with a study by Chelossi et al. (2003), which also recorded a high resistance towards streptomycin and ampicillin in the benthic bacterial community of a marine aquaculture. The prevalence of streptomycin resistance has also been reported in numerous fish and shrimp farms (Dung et al., 2008; Kian Giap et al., 2012; Shah et al., 2014). High incidence of ampicillin resistance has been reported in Vibrio sp. in tropical water (You et al., 2016), and in Enterococci in the recreational water in Malaysia (Dada et al., 2013). In another study conducted by Letchumanan et al. (2015), $82 \%$ of Vibrio parahaemolyticus in retail shrimps in Malaysia displayed a high resistance to ampicillin. This might suggest that high incidences of streptomycin and ampicillin resistance are not restricted to a particular water body, but they are indeed widely distributed in aquatic environments.

A high susceptibility was recorded towards doxycycline, levofloxacin, trimethoprim-sulfamethoxazole and nalidixic acid. From this finding, the high susceptibility of the bacterial isolates towards doxycycline might suggest that doxycycline was not commonly used among fish farmers (Pham et al., 2015). Similarly, high susceptibility of levofloxacin was observed in Vibrio parahaemolyticus isolated from retail shrimps by Letchumanan et al. (2015) and Saifedden et al. (2016). High susceptibility towards trimethoprimsulfamethoxazole was also seen in Aeromonas hydrophila in catfish aquaculture (Paola et al., 1995) and bacteria isolated from sea bass (Bourouni et al., 2000). Esherichia coli isolated from tilapia species in Brazil (Rocha et al., 2014) and Vibrio species isolated from aquaculture water in Sabah (Ransangan et al., 2013) also revealed a high sensitivity towards nalidixic acid. However, in a short period of time, these originally susceptible bacteria may become resistant through acquiring resistance genes via horizontal gene transfer or gene mutations (Allen et al., 2010).

Based on the results of this study, the bacteria were resistant towards a variety of antibiotics, which might be an offshoot of diverse genes that protects them against the therapeutic dose of antibiotics. These genes which are also known as the resistome have the potential to be transferred to pathogens and there has been evidence showing that some clinically relevant resistance genes originated from environmental bacteria (Cattoir et al., 2008). Therefore, bacteria with antibiotic resistance genes get selective advantage over antibiotic-sensitive bacteria in presence of antibiotics, and evidently creates a plethora of resistance patterns exhibited by the aquatic bacteria. The fact that antibiotic resistant bacteria were seen in all five fish farms where no antibiotics were used Further confirmed that resistance genes exist naturally in the environment (Allen et al., 2010).

\section{CONCLUSION}

Based on this study, a high percentage $(74.0 \%$; $n=37 / 50)$ of bacteria found in aquaculture in Sarawak possess MAR indices less than 0.2; suggesting that most isolates have been originated from low antibiotic contaminated areas. However, $40.0 \% \quad(n=20 / 50)$ of the isolates displayed multiple antibiotic resistance which should be of concern, as no known antibiotics were used in these farms. The frequency of bacterial resistance to different classes of antibiotics suggested that those bacteria could be reservoirs for antibiotic resistance genes. There is a need for public authorities to reinforce a systematic management of antibiotics in an aquaculture system. Routine screening of antibiotic resistant bacteria in aquaculture could contribute to a better understanding of the role of aquaculture environment and cultured species in the transmission of MAR among human pathogens. 


\section{ACKNOWLEDGEMENTS}

This study was funded under grant vot No. F07/SpGS/1554/2017.

\section{REFERENCES}

Abdullahi, R., Lihan, S., Carlos, B. S., Bilung, M. K., Mikal, M. K. and Collick, F. (2013). Detection of oprL gene and antibiotic resistance of Pseudomonas aeruginosa from aquaculture environment. European Journal of Experimental Biology 3(6), 148-152.

Allen, H. K., Donato, J., Wang, H. H., Cloud-Hansen, K. A., Davies, J. and Handelsman, J. (2010). Call of the wild: Antibiotic resistance genes in natural environments. Nature Reviews Microbiology 8(4), 251259.

Barton, J. R. and Fløysand, A. (2010). The political ecology of Chilean salmon aquaculture, 1982-2010: A trajectory from economic development to global sustainability. Global Environmental Change 20(4), 739-752.

Bhullar, K., Waglechner, N., Pawlowski, A., Koteva, K., Banks, E. D., Johnston, M. D., Barton H. A. and Wright, G. D. (2012). Antibiotic resistance is prevalent in an isolated cave microbiome. PLOS ONE 7(4), 1-11.

Bostock, J., Mcandrew, B., Richards, R., Jauncey, K., Telfer, T., Lorenzen, K., Little, D., Ross, L., Handisyde, N., Gatward, I. and Corner, R. (2010). Aquaculture: Global status and trends. Philosophical Transactions of The Royal Society B (365), 28972912.

Bourouni, O. C., Bour, M. El, Calo-mata, P. and Barros-velàzquez, J. (2000). Antimicrobial resistance and potential probiotic application of Enterococcus spp. in Sea Bass and Sea Bream aquaculture. In: Antibiotic Resistant Bacteria - A Continuous Challenge in the New Millennium. Marina, P. (ed.). InTech, Croatia. pp. 513-530.

Bush, K., Courvalin, P., Dantas, G., Davies, J., Eisenstein, B., Huovinen, P., et al. (2011). Tackling antibiotic resistance. Nature Reviews Microbiology 9(12), 894-896.

Cabello, F. C., Godfrey, H. P., Tomova, A., Ivanova, L., Dölz, H., Millanao, A. and Buschmann, A. H. (2013). Antimicrobial use in aquaculture re-examined: Its relevance to antimicrobial resistance and to animal and human health. Environmental Microbiology 15(7), 1917-1942.

Cantas, L., Shah, S. Q. A., Cavaco, L. M., Manaia, C. M., Walsh, F., Popowska, M., Garelick, H., Bürgmann, H. and Sørum, H. (2013). A brief multidisciplinary review on antimicrobial resistance in medicine and its linkage to the global environmental microbiota. Frontiers in Microbiology 4, 1-14.

Cattoir, V., Poirel, L., Aubert, C., Soussy, C. J. and Nordmann, P. (2008). Unexpected occurrence of plasmid-mediated quinolone resistance determinants in environmental Aeromonas spp. Emerging Infectious Diseases 14(2), 231-237.
Chamosa, L. S., Álvarez, V. E., Nardelli, M., Quiroga, M. P., Cassini, M. H. and Centrón, D. (2017). Lateral antimicrobial resistance genetic transfer is active in the open environment. Scientific Reports 7(1), 1-12.

Chelossi, E., Vezzulli, L., Milano, A., Branzoni, M., Fabiano, M., Riccardi, G. and Banat, I. M. (2003). Antibiotic resistance of benthic bacteria in fish-farm and control sediments of the Western Mediterranean. Aquaculture 219(4), 83-97.

CLSI. (2017). Performance standards for antimicrobial susceptibility testing. CLSI, Pennsylvania, USA.

Dada, A. C., Ahmad, A., Usup, G., Heng, L. Y. and Hamid, R. (2013). Speciation and antimicrobial resistance of Enterococci isolated from recreational beaches in Malaysia. Environmental Monitoring and Assessment 185(9), 7427-7443.

Deng, Y., Wu, Y., Jiang, L., Tan, A., Zhang, R. and Luo, L. (2016). Multi-drug resistance mediated by class 1 integrons in Aeromonas isolated from farmed freshwater animals, 7, 1-7.

Department of Statistics Malaysia. (2018). Selected agricultural indicators, Malaysia, 2018. https://www.dosm.gov.my [Retrieved on 26 July 2019].

Done, H. Y., Venkatesan, A. K. and Halden, R. U. (2015). Does the recent growth of aquaculture create antibiotic resistance threats different from those associated with land animal production in agriculture? The American Association of Pharmaceutical Scientists Journal 17(3), 513-524.

Dung, T. T., Haesebrouck, F., Tuan, N. A., Sorgeloos, P., Baele, M. and Decostere, A. (2008). Antimicrobial susceptibility pattern of Edwardsiella ictaluri isolates from natural outbreaks of bacillary necrosis of Pangasianodon hypophthalmus in Vietnam. Microbial Drug Resistance 14(4), 311-316.

Farith, K., Awang Ahmad, A. H., Samuel, L., Mohd Hasnain, M. H. and Hairul Azman, R. (2015). In vitro antagonism of Phytophthora capsici and Fusarium solani by bacterial isolates from Sarawak. Malaysian Journal of Microbiology 11(2), 137-143.

Heuer, O. E., Kruse, H., Grave, K., Collignon, P., Karunasagar, I. and Angulo, F. J. (2009). Human health consequences of use of antimicrobial agents in aquaculture. Clinical Infectious Diseases 49(8), 12481253.

Huys, G. (2003). Sampling and Sample Processing Procedures for the Isolation of AquacultureAssociated Bacteria. Standard Operating Procedures. Belgium: Ghent University, Belgium.

Iñiguez-Palomares, C., Pérez-Morales, R. and AcedoFélix, E. (2007). Evaluation of probiotic properties in Lactobacillus isolated from small intestine of piglets. Revista Latinoamericana de Microbiologia, 49(3-4), 46-54.

Kathleen, M. M., Samuel, L., Felecia, C., Reagan, E. L., Kasing, A., Lesley, M. and Seng Chiew, T. (2016). Antibiotic resistance of diverse bacteria from aquaculture in Borneo. International Journal of Microbiology 2016, 1-9. 
Kian Giap, Y., Chui Wei, B. and Choon Weng, L. (2012). Antimicrobial resistance in bacteria isolated from tropical coastal waters of Peninsular Malaysia. Malaysian Journal of Science 31(2), 111-120.

Krumperman, P. H. (1983). Multiple antibiotic resistance indexing of Escherichia coli to identify high-risk sources of fecal contamination of foods. Applied and Environmental Microbiology 46(1), 165-170.

Kui Soon, L., Samuel, L., Dasthagir, F. F. G., Kathleen, M., Felicia, C. and Kok Hua, Ng. (2014). Microbiological and physicochemical analysis of water from empurau fish (Tor Tambroides) farm in Kuching, Sarawak, Malaysian Borneo. International Journal of Scientific and Technology Research 3(6), 285-292.

Lesley, M. B., Sin Chai, L., Ahmad Syatir, T., Kian Ted, C. and Kasing A. (2018). Prevalence, genetic heterogeneity, and antibiotic resistance profile of Listeria spp. and Listeria monocytogenes at farm Level: A highlight of ERIC- and BOX-PCR to reveal genetic diversity. BioMed Research International 2018, 1-12.

Lesley, M. B., Velnetti, L., Cheah, Y. K., Son, R., Kasing, A., Samuel, L., Micky, V. and Nishibuchi, M. (2011). Antibiotic resistance and plasmid profiling of Vibrio parahaemolyticus isolated from cockles (Anadara granosa) at Tanjung Karang, Kuala Selangor. International Food Research Journal 18(3), 1183-1188.

Letchumanan, V., Yin, W. F., Lee, L. H. and Chan, K. G. (2015). Prevalence and antimicrobial susceptibility of Vibrio parahaemolyticus isolated from retail shrimps in Malaysia. Frontiers in Microbiology 6(Jan), 1-11.

Ng, K. H., Samuel, L., Kathleen, M. M., Leong, S. S. and Felecia, C. (2014). Distribution and prevalence of chloramphenicol-resistance gene in Escherichia coli isolated from aquaculture and other environment. International Food Research Journal 21(4), 13211325.

Paola, A. D. E., Paola, A. D. E., Peeler, J. T., Peeler, J. T., Rodrick, G. E. and Rodrick, G. E. (1995). Effect of oxytetracycline-medicated feed on antibiotic resistance of Gram-negative bacteria in cat fish ponds. Microbiology 61(6), 2335-2340.

Patil, H. J., Benet-Perelberg, A., Naor, A., Smirnov, M., Ofek, T., Nasser, A., Minz, D. and Cytryn, E. (2016). Evidence of increased antibiotic resistance in phylogenetically-diverse Aeromonas isolates from semi-intensive fish ponds treated with antibiotics. Frontiers in Microbiology 7(Nov), 1-12.

Paulson, J. R., Mahmoud, I. Y., Al-Musharafi, S. K. and Al-Bahry, S. N. (2016). Antibiotic resistant bacteria in the environment as bio-indicators of pollution. The Open Biotechnology Journal 10(Suppl-2, M7), 342351.

Perry, J. A. and Wright, G. D. (2013). The antibiotic resistance "mobilome": Searching for the link between environment and clinic. Frontiers in Microbiology 4(May), 1-7.

Pham, D. K., Chu, J., Do, N. T., Brose, F., Degand, G., Delahaut, P., De Pauw, E., Douny, C., Van Nguyen,
K., Vu, T. D., Scippo, M. L. and Wertheim, H. F. L. (2015). Monitoring antibiotic use and residue in freshwater aquaculture for domestic use in Vietnam. EcoHealth 12(3), 480-489.

Phillips, I., Casewell, M., Cox, T., De Groot, B., Friis, C., Jones, R., Nightingale, C., Preston, R. and Waddell, J. (2004). Does the use of antibiotics in food animals pose a risk to human health? A critical review of published data. Journal of Antimicrobial Chemotherapy 53(1), 28-52.

Radhouani, H., Silva, N., Poeta, P., Torres, C., Correia, S. and Igrejas, G. (2014). Potential impact of antimicrobial resistance in wildlife, environment, and human health. Frontiers in Microbiology 5(Feb), 1-12.

Ransangan, J., Khor, L., Imm, L., Lal, T. M. and Sade, A. (2013). Phenotypic characterization and antibiotic susceptibility of Vibrio spp. isolated from aquaculture waters on the west coast of Sabah, Malaysia. International Journal of Research in Pure and Applied Microbiology 3(3), 58-66.

Rocha, R. D. S., Leite, L. O., de Sousa, O. V. and Vieira, R. H. (2014). Antimicrobial susceptibility of Escherichia coli isolated from fresh-marketed Nile Tilapia (Oreochromis niloticus). Journal of Pathogens 2014(c), 756539.

Romero, J., Gloria, C. and Navarrete, P. (2012). Antibiotics in aquaculture-Use, abuse and alternatives. In: Health and Environment in Aquaculture. Carvalho, E., David, G. S. and Silva, R. J. (eds.). IntechOpen, Austria. pp. 160-198.

Samuel, L., Marian, M. M., Apun, K., Lesley, M. B. and Son, R. (2011). Characterization of Escherichia coli isolated from cultured catfish by antibiotic resistance and RAPD analysis. International Food Research Journal 18(3), 971-976.

Samuel, L., Keong Choon, Y., Kok Hua, N. and Mohd Effendi, W. (2014a). Screening for antimicrobial activity of fungi in soil samples collected from Kubah National. International Journal of Scientific and Technology 3(2), 1-9.

Samuel, L., Sin Lin, C., Ismail, A., Fazia, M. S., Kok Hua, N. and Awang, A. S. (2014b). Antimicrobial producing microbes isolated from soil samples collected from Nanga Merit Forest in Sarawak, Malaysian Borneo. European Journal of Experimental Biology 4(1), 494-501.

Saifedden, G., Farinazleen, G., Nor-Khaizura, A., Kayali, A., Nakaguchi, Y., Nishibuchi, M. and Son, R. (2016). Antibiotic susceptibility profile of Vibrio parahaemolyticus isolated from shrimp in Selangor, Malaysia. International Food Research Journal 23(Dec), 2732-2736.

Schmidt, A., Bruun, M., Dalsgaard, I., Pedersen, K. and Larsen, J. (2000). Occurrence of antimicrobial resistance in fish-pathogenic and environmental bacteria associated with four danish rainbow trout farms. Applied and Environmental Microbiology 66(11), 4908-4915.

Seng Chiew, T., Samuel, L., Khar Mun, S., Natalia, U., Lay Ching, C. and Müller, M. (2018). Screening and 
Malays. J. Microbiol. Vol 16(4) 2020, pp. 263-274

DOI: http://dx.doi.org/10.21161/mjm.190522

characterisation of two strains of Pseudomonas aeruginosa from aquaculture and water environment. Malaysian Journal of Microbiology 14(2), 329-334.

Shah, S. Q. A., Cabello, F. C., L'Abée-Lund, T. M., Tomova, A., Godfrey, H. P., Buschmann, A. H. and Sørum, H. (2014). Antimicrobial resistance and antimicrobial resistance genes in marine bacteria from salmon aquaculture and non-aquaculture sites. Environmental Microbiology 16(5), 1310-1320.

Singh, S. B. (2014). Confronting the challenges of discovery of novel antibacterial agents. Bioorganic and Medicinal Chemistry Letters 24(16), 3683-3689.

Smith, P. (2008). Antimicrobial resistance in aquaculture. Revue Ssientifique et Technique (International Office of Epizootics) 27(1), 243-64.

Swamy, M. K. and Rudramurthy, G. R. (2016). Antimicrobial agents: Current status and future challenges. Austin Pharmacology and Pharmaceutics 1(1), 1004.

Tanil, G. B., Radu, S., Nishibuchi, M., Rahim, R. A., Napis, S., Maurice, L. and Gunsalam, J. W. (2005). Characterization of Vibrio parahaemolyticus isolated from coastal seawater in peninsular Malaysia. Southeast Asian Journal of Tropical Medicine and Public Health 36(4), 940-945.

Watts, J., Schreier, H., Lanska, L. and Hale, M. (2017). The rising tide of antimicrobial resistance in aquaculture: Sources, sinks and solutions. Marine Drugs 15(6), 158.

You, K. G., Bong, C. W. and Lee, C. W. (2016). Antibiotic resistance and plasmid profiling of Vibrio spp. in tropical waters of Peninsular Malaysia. Environmental Monitoring and Assessment 188(3), 171. 